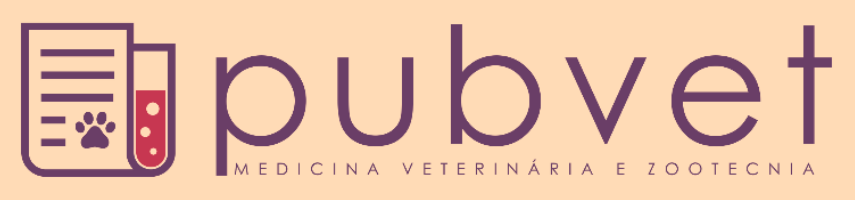

https://doi.org/10.31533/pubvet.v13n8a394.1-6

\title{
Efeito alelopático do extrato de folhas de Pequi e Cagaita na germinação de sementes de Tamboril
}

\author{
Weuler Alves Vasconcelos ${ }^{1^{*}} \bullet$, Ana Clara Alencar Lambert ${ }^{2} \bullet$, Sabrina do Couto de \\ Miranda $^{30}$, Priscila Bezerra Souza $^{4}{ }^{\circ}$, Sybelle Barreira ${ }^{5}$ \\ ${ }^{1}$ Mestrando na Universidade Federal do Tocantins, Programa de Pós-graduação em Ciências Florestais e Ambientais, Gurupi-TO, Brasil. \\ ${ }^{2}$ Engenheira Florestal na Gerência de Recursos Naturais do Instituto Brasileiro de Geografia e Estatística, Florianópolis - SC, Brasil \\ ${ }^{3}$ Professora da Universidade Estadual de Goiás, Departamento de Botânica, Palmeiras de Goiás - GO, Brasil. \\ ${ }^{4}$ Professora da Universidade Federal do Tocantins, Programa de Pós-graduação em Ciências Florestais e Ambientais, Gurupi-TO, Brasil. \\ ${ }^{5}$ Professora da Universidade Federal de Goiás, Departamento de Engenharia Florestal, Goiânia - GO, Brasil. \\ *Autor para correspondência. E-mail: weulervasconcelos@hotmail.com
}

Resumo. A interação em florestas naturais envolve vários fatores ecológicos, desde a saúde da árvore matriz na produção de propágulos até o impedimento da germinação de determinadas espécies por meio de compostos alelopáticos. Dessa forma o objetivo deste estudo foi avaliar o efeito alelopático do extrato hidroalcoólico de folhas de Caryocar brasiliense e Eugenia dysenterica na germinação de sementes de Enterolobium contortisiliquum. Foi realizado teste de germinação em delineamento inteiramente casualizado, com quatro repetições de 25 sementes cada, sendo os tratamentos: $\mathrm{T} 1=$ testemunha (água destilada); T2 $=E$. dysenterica na concentração de $0,75 \% ; \mathrm{T} 3=E$. dysenterica na concentração de $1,5 \%$; T4 $=C$. brasiliense na concentração de $0,75 \%$; T5 $=$ C. brasiliense na concentração de $1,5 \%$. Foram avaliadas a taxa de germinação, o índice de velocidade de germinação, assim como o número de plântulas normais, anormais e mortas. Os resultados apontaram que os extratos de Cagaita nas duas concentrações e de Pequi na menor concentração influenciaram negativamente no processo germinativo. $\mathrm{O}$ extrato de Pequi na concentração de $1,5 \%$ foi o responsável pelo maior número de plântulas anormais. No sexto dia foi possível verificar maior diferença no índice de velocidade de germinação entre os tratamentos.

Palavras chave: aleloquímicos, Cerrado, sementes florestais

\section{Allelopathic effect of Pequi and Cagaita leaf extract on Tamboril seed germination}

Abstract. The interaction in natural forests involves various ecological factors, since the array tree health in the production of propagating material to preventing germination of certain species by means of alelopáticos compounds. Thus, the objective of this study was to evaluate the allelopathic effect of hydroalcoholic extract of leaves of Caryocar brasiliense and Eugenia dysenterica on seed germination of Enterolobium contortisiliquum. Germination test was performed in completely randomized design, with four replicates of 25 seeds each, being the treatments: $\mathrm{T} 1=$ witness (distilled water); $\mathrm{T} 2=$ E. dysenterica in the concentration of $0.75 \%$; $\mathrm{T} 3=E$. dysenterica in the concentration of $1.5 \% ; \mathrm{T} 4=C$. brasiliense on concentration of $0.75 \%$; $\mathrm{T} 5=C$. brasiliense on concentration of $1.5 \%$. We evaluated the germination rate, germination speed index, as well as the number of normal, abnormal seedlings and dead. The results showed that the Cagaita extracts in two concentrations and of Pequi in lower concentration influenced negatively in the germinal process. The Pequi extract at a concentration of $1.5 \%$ was responsible for the 
largest number of abnormal seedlings. On the sixth day, it was possible to check larger difference in the germination speed index among the treatments.

Keywords: allelochemicals, Brazilian Savanna, forest seeds

\section{Efecto alelopático del extracto de hoja de Pequi y Cagaita sobre la germinación de semillas de Tamboril}

Resumen. La interacción en los bosques naturales implica varios factores ecológicos, desde la salud del árbol matriz en la producción de materiales hasta el impedimento de la germinación de algunas especies por medio de compuestos alelopáticos. Así el objetivo de este estudio fue evaluar el efecto alelopático del extracto hidroalcohólico de hojas de Caryocar brasiliense y Eugenia dysenterica en la germinación de la semilla de Enterolobium contortisiliquum. La prueba de germinación se realizó en diseño completamente al azar, con cuatro repeticiones de 25 semillas cada una, siendo los tratamientos: $\mathrm{T} 1=$ testigo (agua destilada); $\mathrm{T} 2=E$. dysenterica en la concentración de $0,75 \%$; T3 $=E$. dysenterica en la concentración de $1,5 \%$; $\mathrm{T} 4=C$. brasiliense en concentración de $0,75 \%$; T5 $=C$. brasiliense sobre concentración del 1,5\%. Se evaluó la tasa de germinación, índice de velocidad de germinación, así como el número de plántulas normales, anormales y muertas. Los resultados mostraron que los extracto de $E$. dysenterica en dos concentraciones y del $C$. brasiliense en menor concentración influenciaron negativamente en el proceso germinativo. El extracto de $C$. brasiliense a una concentración del $1,5 \%$ fue responsable por el mayor número de plántulas anormales. En el sexto día fue posible comprobar la mayor diferencia en el índice de velocidad de germinación entre los tratamientos.

Palabras clave: aleloquímicos, Cerrado, semillas forestales

\section{Introdução}

Enterolobium contortisiliquum (Vell.) Morong (Tamboril) é uma espécie com ocorrência em todos os biomas brasileiros, exceto bioma Amazônico (Reflora, 2018). É uma espécie pertencente à família Fabaceae, podendo atingir até 35 metros com perda de folhas durante o inverno (Lorenzi et al., 2002). As sementes desta espécie apresentam tegumento rígido impossibilitando a germinação sem prétratamento (Melo Junior et al., 2018). Devido a sua ampla distribuição, nos ambientes naturais a espécie interage com diversas outras espécies de diversas formas, dentre as quais o Caryocar brasiliense Cambess (Pequi) e Eugenia dysenterica DC. (Cagaita) motivo pelo qual foram o objeto de desse estudo visto sua ampla distribuição em áreas de Cerrado (Ratter et al., 2003). O Caryocar brasiliense é uma das 38 espécies amplamente distribuídas no Cerrado, ocorrendo em $61 \%$ das 316 áreas estudadas por Ratter et al. (2003). Já a Eugenia dysenterica ocorreu em 38,3\% das áreas e é indicadora de solos mesotróficos. Mendonça et al. (2008) afirmam que as duas espécies estão associadas as mais diversas fitofisionomias do bioma Cerrado.

Visto que o Pequi, a Cagaita e o Tamboril ocorrem com facilidade em vários ambientes do Cerrado, os mesmos podem interagir de forma natural entre si. Uma forma de interação é a ação alelopática, definida como o efeito positivo ou negativo entre plantas por meio de substâncias químicas, chamadas de aleloquímicos (Ferreira et al., 2010). Essas substâncias estão presentes em diversas espécies em seus diferentes órgãos (Alves et al., 2004). A interação entre espécies em um ambiente pode ser fortemente pressionada e resultar em alterações na estrutura e dinâmica de uma comunidade, sendo a alelopatia responsável por tais interações e adotada como um mecanismo ecológico (Chou, 1999; Oliveira et al., 2011).

Dessa forma, objetivou-se avaliar o efeito alelopático do extrato hidroalcoólico de folhas Pequi e Cagaita na germinação de sementes de Tamboril.

\section{Material e métodos}

O trabalho foi realizado no Laboratório de Reprodução de Espécies Florestais (REFLOR) da Universidade Federal de Goiás, Campus Samambaia. As sementes de tamboril foram coletadas em uma única matriz, localizada sob as coordenadas $16^{\circ} 36^{\prime} 54,77^{\prime \prime} \mathrm{S}$ e $49^{\circ} 17^{\prime} 6,82^{\prime}$ 'O. 
Para o preparo do extrato hidroalcoólico foram utilizadas folhas verdes do terço basal de vários ramos de um indivíduo de Cagaita e outro de Pequi selecionados aleatoriamente em um fragmento restaurado de Cerrado, denominado Cinturão Verde, localizado na Escola de Agronomia da UFG, presentes nas

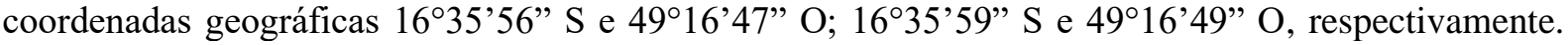
Para a escolha das folhas utilizou-se o critério visual, ou seja, sem sinal de doença ou anomalias. As mesmas após coletas foram secas com auxílio de estufa de circulação forçada por 24 horas a $50^{\circ} \mathrm{C}$, posteriormente foram trituradas com auxílio de cadinho.

O material obtido a partir desse procedimento foi pesado e separado $15 \mathrm{~g}$ do mesmo, por espécie, que foi misturado a $980 \mathrm{~mL}$ de água destilada e $20 \mathrm{~mL}$ de álcool (97\%), sendo agitado para homogeneização. Essa mistura foi preparada em um erlenmeyer que teve sua boca vedada com papel insulfilm e reservado por 24 horas em geladeira comum, com agitações esporádicas, adaptando assim o que foi realizado por Aires (2007). Logo, foi obtido o extrato aquoso de cada espécie na concentração de $1,5 \%$. Para a concentração de $0,75 \%$, o processo foi a partir da diluição simples pelo composto mais concentrado. Para a superação de dormência tegumentar das sementes de Tamboril foi realizado o método da escarificação mecânica (Pereira et al., 2014), no qual as sementes foram colocadas em contato com a superfície áspera de uma lixa mecânica do tipo 6" o que proporcionou uma abertura, porém sem danos ao embrião, para facilitar a absorção de água e iniciar o processo de germinação.

Para a realização dos bioensaios foi utilizado o delineamento experimental inteiramente casualizado (DIC), composto com cinco tratamentos, sendo: $\mathrm{T} 1=$ testemunha (água destilada); $\mathrm{T} 2=$ E. dysenterica na concentração de $0,75 \%$; T3 $=E$. dysenterica na concentração de $1,5 \%$; T4 $=C$. brasiliense na concentração de $0,75 \%$; T5 $=C$. brasiliense na concentração de $1,5 \%$, com quatro repetições de 25 sementes cada. Os tratamentos foram dispostos em caixas gerbox com duas folhas de papel mata borrão umedecido com $20 \mathrm{~mL}$ do extrato, de acordo com sua concentração, para cada repetição. As mesmas foram dispostas em um germinador de sementes do tipo Mangelsdorf, por 15 dias a temperatura de $25^{\circ}$ $\mathrm{C}$ e luminosidade natural. A reposição da quantidade de extrato na caixa gerbox foi realizada diariamente durante o período do experimento colocando $10 \mathrm{~mL}$ do extrato em seus respectivos tratamentos.

Os parâmetros avaliados foram a porcentagem de germinação final aos 15 dias (PG \%) e o índice de velocidade de germinação (IVG), sendo que foram consideradas sementes germinadas aquelas com emissão do primeiro par de folhas e emissão radicular superior a um centímetro. Ainda, analisou-se o percentual de plântulas normais, anormais e mortas de acordo com os parâmetros proposto por (Santos et al., 2013).

Os dados foram submetidos à análise de normalidade, posteriormente a análise de variância (ANOVA) e as médias foram comparadas pelo teste de Tukey, a 5\% de probabilidade, ambos os testes realizados com auxílio do programa Assitat versão 7.7.

\section{Resultados e discussão}

Os extratos hidroalcoólicos testados de $C$. brasiliense (Pequi) e $E$. dysenterica (Cagaita) tiveram seus respectivos $\mathrm{pH}$ medidos a temperatura ambiente após finalizados. As características ácidas das soluções já eram esperadas visto que são oriundas de espécies típicas do Cerrado, no qual apresentam plantas e solos com características oligotroficas e xeromórficas (Tabela 1). Gatti et al. (2004) e Maraschin-Silva \& Aqüila (2005) trabalhando com efeito alelopático, também mediram o pH de suas soluções para excluir efeitos adversos do mesmo.

Tabela 1. Valores do pH das soluções hidroalcoólicas medidos a temperatura ambiente

\begin{tabular}{llcc}
\hline Tratamento & Solução hidroalcoólicas & Concentração, \% & $\mathrm{pH}\left(24^{\circ} \mathrm{C}\right)$ \\
\hline $\mathrm{T} 1=$ & Testemunha (água destilada) & 0 & 4,7 \\
$\mathrm{~T} 2$ & E. dysenterica & 0,75 & 1,85 \\
$\mathrm{~T} 3$ & E. dysenterica & 1,5 & 1,77 \\
$\mathrm{~T} 4$ & C. brasiliense & 0,75 & 2,09 \\
$\mathrm{~T} 5$ & C. brasiliense & 1,5 & 1,52 \\
\hline
\end{tabular}


Para a germinação, os tratamentos com extrato de Cagaita e de Pequi na menor concentração diferiram estatisticamente da testemunha, assim como o tratamento com Cagaita em maior concentração. Harun et al. (2014) explicam que isso ocorre por os aleloquímicos liberados são inibidores da germinação, do crescimento e da fisiologia, pois interferem em vários processos fisiológicos. Na Tabela 2 apresenta a comparação das médias dos tratamentos através do Teste de Tukey a 5\% de probabilidade para a variável porcentagem de germinação e o IVG por tratamento calculado.

Tabela 2. Valores médios da porcentagem de germinação e do índice de velocidade de germinação (IVG) das sementes de Enterolobium contortisiliquum (Vell.) Morong, submetidas as diferentes diluições dos extratos de Eugenia dysenterica DC e Caryocar brasiliense Cambess

\begin{tabular}{lcc}
\hline Tratamentos & Germinação (\%) & IVG \\
\hline Testemunha & $92 \mathrm{~A}$ & $6,95 \mathrm{~A}$ \\
Cagaita na concentração de $0,75 \%$ & $72 \mathrm{~B}$ & $4,82 \mathrm{~B}$ \\
Cagaita na concentração de 1,5\% & $73 \mathrm{~B}$ & $5,93 \mathrm{~B}$ \\
Pequi na concentração de $0,75 \%$ & $72 \mathrm{~B}$ & $5,04 \mathrm{~B}$ \\
Pequi na concentração de 1,5\% & $78 \mathrm{AB}$ & $5,03 \mathrm{~B}$ \\
\hline CV $(\%)$ & 11,02 & 16,02 \\
\hline
\end{tabular}

Allem et al. (2014) observaram o efeito alelopático e a fitoxidez provocada por folhas de Pequi quando incorporadas ao substrato, influenciando negativamente na germinação de sementes de sorgo, gergelim, braquiária, e de ipê-roxo. Tais resultados corroboram com o que foi encontrado nesse trabalho. Reichel et al. (2013) afirmam que as propriedades biológicas das substâncias alelopáticas podem ser alteradas, assim como seus efeitos, e que os fatores determinantes para essa mudança podem ser a fonte do extrato, a forma de obtenção, a concentração e as condições climáticas a qual estão expostas, justificando assim as diferentes formas de interação natural entre os aleloquímicos liberados e as sementes no meio natural, e a forma que o extrato de Pequi na maior concentração reagiu no estudo.

A curva do Índice de Valor de Germinação (IVG) demostrou um pico de germinação inicial para o tratamento com extrato de Cagaita na concentração de 1,5\%, germinando antes mesmo do tratamento testemunha, entretanto não se pode afirmar que o extrato influenciou no resultado, visto que o processo de germinação utiliza prioritariamente as reservas da semente e a germinação inicial é menos afetada pela presença de aleloquímicos provenientes de extratos aplicados em bioensaios (Jacobi \& Ferreira, 1991; Miró et al., 1998). Em estudo conduzido por Borella et al. (2009), o IVG foi afetado em ambos os bioensaios, com reduções no número médio de sementes de alface germinadas por dia em relação ao aumento da concentração dos extratos, corroborando parcialmente com este estudo.

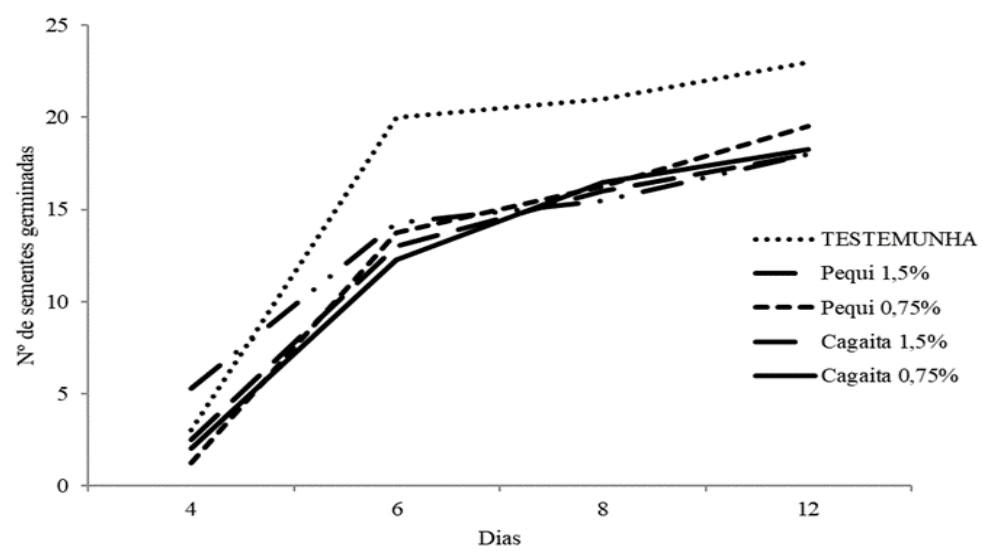

Figura 1. Número de sementes germinadas de Enterolobium contortisiliquum (Vell.) Morong (Tamboril) ao decorrer dos dias por tratamento (IVG).

Jefferson \& Pennacchio (2003) afirmam que as sementes que germinam mais lentamente podem dar origem a plântulas com tamanho reduzido ou ainda com anormalidades. Aplicando os parâmetros 
estipulados por (Santos et al., 2013) obteve-se os resultados para o número de plântulas normais, anormais e mortas (Figura 2).

O maior número de plântulas anormais foi verificado no bioensaio com Pequi na maior concentração, entretanto, a média de germinação desse tratamento não diferiu significativamente da testemunha, mostrando que o mesmo proporciona plântulas de menor qualidade visual, entretanto não inibe totalmente sua germinação. Plântulas anormais e mortas em bioensaios que verificam o potencial alelopático é esperado, visto que os compostos liberados influenciam no desenvolvimento da parte aérea ou da parte radicular, formando plantas de qualidade inferior.

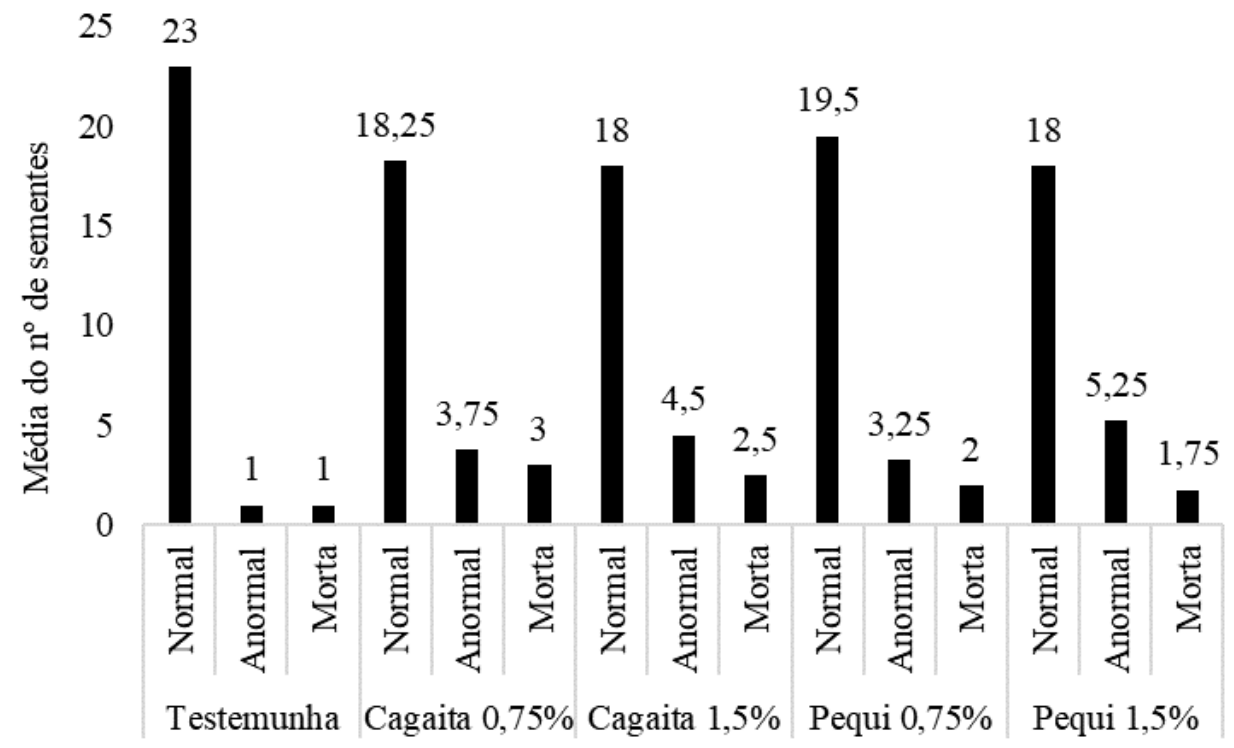

Figura 2. Média do número de plântulas de Enterolobium contortisiliquum (Vell.) Morong (Tamboril) normais, anormais e mortas por tratamento.

Estudos buscando entender a interação entre espécies do Cerrado são importantes, visto a necessidade de se recuperar áreas de forma mais natural possível, formulado assim modelos que levem em consideração a escolha de espécies para recomposição que não liberem aleloquímicos no meio que possam a vir influenciar negativamente na germinação de outras espécies.

\section{Conclusões}

O extrato hidroalcoólico de E. dysenterica, nas duas concentrações, e o de $C$. brasiliense, na menor concentração, foram os que mais influenciaram na germinação de E. contortisiliquum, diminuindo a taxa de germinação significativamente. O bioensaio com C. brasiliense na concentração de $1.5 \%$ apresentou maior número de plântulas anormais. São necessários estudos de campo, com ensaio em viveiros para saber com maior exatidão os efeitos das folhas de Pequi e Cagaita na germinação de Tamboril.

\section{Referências bibliográficas}

Aires, S. S. Potencial alelopático de espécies nativas do Cerrado na germinação e desenvolvimento inicial de invasoras. 2007. 61p. Dissertação (Mestrado em Botânica) - Universidade de Brasília, Brasília.

Allem, L. N., Gomes, A. S. \& Borghetti, F. (2014). Pequi leaves incorporated into the soil reduce the initial growth of cultivated, invasive and native species. Anais da Academia Brasileira de Ciências, 86(4):1761-1768.

Alves, M. C. S., Medeiros Filho, S., Innecco, R. \& Torres, S. B. (2004). Alelopatia de extratos voláteis na germinação de sementes e no comprimento da raiz de alface. Pesquisa Agropecuária Brasileira, 39(11):1083-1086. 
Borella, J., Wandscheer, A. C. D., Bonatti, L. C. \& Pastorini, L. H. (2009). Efeito alelopático de extratos aquosos de Persea americana Mill. sobre Lactuca sativa L. Revista Brasileira de Biociências, 7(3).

Chou, C.-H. (1999). Roles of allelopathy in plant biodiversity and sustainable agriculture. Critical Reviews in Plant Sciences, 18(5):609-636.

Ferreira, E. G. B. S., Matos, V. P., Sena, L. H. M. \& Sales, A. G. d. F. A. (2010). Efeito alelopático do extrato aquoso de sabiá na germinação de sementes de fava. Revista Ciência Agronômica, 41(3):463467.

Gatti, A. B., Perez, S. C. J. G. \& Lima, M. I. S. (2004). Atividade alelopática de extratos aquosos de Aristolochia esperanzae O. Kuntze na germinação e no crescimento de Lactuca sativa L. e Raphanus sativus L. Acta Botanica Brasilica, 18(3):459-472.

Harun, M. A. Y. A., Johnson, R. W. R. \& Uddin, M. Z. (2014). Allelopathic potential of invasion processes. South African Journal of Botany, 93157-166.

Jacobi, U. S. \& Ferreira, A. G. (1991). Efeitos alelopáticos de Mimosa bimucronata (DC) OK. sobre espécies cultivadas. Pesquisa Agropecuária Brasileira, 26(7):935-943.

Jefferson, L. V. \& Pennacchio, M. (2003). Allelopathic effects of foliage extracts from four Chenopodiaceae species on seed germination. Journal of Arid Environments, 55(2):275-285.

Lorenzi, H., Matos, F. J. \& Francisco, J. M. (2002). Plantas medicinais no Brasil: nativas e exóticas (Vol. 1).

Maraschin-Silva, F. \& Aqüila, M. E. A. (2005). Potencial alelopático de Dodonaea viscosa (L.) Jacq. Iheringia. Série Botânica., 60(1):92-98.

Melo Junior, J. L. A., Melo, L. D. F. A., Araujo Neto, J. C. \& Ferreira, V. M. (2018). Germination and morphology of seeds and seedlings of Colubrina glandulosa Perkins after overcoming dormancy. Australian Journal of Crop Science, 12(4):639-647.

Mendonça, R. C., Felfili, J. M., Walter, B. M. T., Silva-Júnior, M. d., Rezende, A. V., Filgueiras, T. d. S., . . . Fagg, C. W. (2008). Flora vascular do bioma Cerrado: checklist com 12.356 espécies. Cerrado: Ecologia e Flora, 2422-442.

Miró, C. P., Ferreira, A. G. \& Aquila, M. E. A. (1998). Alelopatia de frutos de erva-mate (Ilex paraguariensis) no desenvolvimento do milho. Pesquisa Agropecuária Brasileira, 33(8):1261-1270.

Oliveira, L. G. A., Belinelo, V. J., Almeida, M. S., Aguilar, E. B. \& Vieira Filho, S. A. (2011). Alelopatia de Emilia sonchifolia (L.) DC.(Asteraceae) na germinação e crescimento inicial de sorgo, pepino e picão preto. Enciclopédia Biosfera, 7(12):1-10.

Pereira, V. J., Santana, D. G., Lobo, G. A., Brandão, N. A. L. \& Soares, D. C. P. (2014). Eficiência dos tratamentos para a superação ou quebra de dormência de sementes de Fabaceae. Revista de Ciências Agrárias, 37(2):187-197.

Ratter, J. A., Bridgewater, S. \& Ribeiro, J. F. (2003). Analysis of the floristic composition of the Brazilian Cerrado vegetation III: comparison of the woody vegetation of 376 areas. Edinburgh Journal of Botany, 60(1):57-109.

REFLORA. Flora do Brasil 2020 em construção. Rio de Janeiro: Jardim Botânico. Disponível em: <http://floradobrasil.jbrj.gov.br/>. Acesso em: 27 nov. 2018.

Reichel, T., Barazetti, J. F., Stefanello, S., Paulert, R. \& Zonetti, P. C. (2013). Allelopathy of leaf extracts of jatropha (Jatropha curcas L.) in the initial development of wheat (Triticum aestivum L.). IDESIA, 31(1):45-52.

Santos, C. A. C., Vieira, E. L., Peixoto, C. P. \& Ledo, C. A. S. (2013). Germinação de sementes e vigor de plântulas de maracujazeiro amarelo submetidos à ação do ácido giberélico. Bioscience Journal, 29(2):400-407.

Recebido: 10 de julho, 2019.

Aprovado: 26 de agosto, 2019.

Publicado: 17 de setembro, 2019.

Licenciamento: Este artigo é publicado na modalidade Acesso Aberto sob a licença Creative Commons Atribuição 4.0 (CC-BY 4.0), a qual permite uso irrestrito, distribuição, reprodução em qualquer meio, desde que o autor e a fonte sejam devidamente creditados. 